\title{
Caractéristiques botaniques et phytochimiques de Vernonia kotschyana Sch. Bip. ex Walp. mise en culture et utilisée dans le traitement des gastrites et l'ulcère gastroduodénal au Mali
}

\author{
Mamadou Lamine DIARRA ${ }^{1,2^{*}}$, Adama DENOU ${ }^{2}$, Bréhima Lamine COULIBALY ${ }^{4}$, \\ Adiaratou TOGOLA ${ }^{2,3}$, Djibril SANOGO ${ }^{4}$, Rokia SANOGO ${ }^{2,3}$, Mamoudou TRAORE $^{4}$, \\ Drissa DIALLO ${ }^{2,3}$ et Kandioura NOBA ${ }^{1}$ \\ ${ }^{I}$ Laboratoire de Botanique et Biodiversité, Université Cheikh Anta Diop de Dakar, \\ B.P.5005, Dakar Fann, Sénégal. \\ ${ }^{2}$ Faculté de Pharmacie, Université des Sciences, des Techniques et des Technologies de Bamako, \\ BP.1805, Bamako, Mali. \\ ${ }^{3}$ Département de Médecine Traditionnelle (DMT), Institut National de Recherche en Santé Publique (INRSP), \\ BP.1746, Bamako, Mali. \\ ${ }^{4}$ Laboratoire d'Ethnobotanique, Institut Polytechnique Rural de Formation et de Recherche Appliquée \\ (IPR/IFRA) de Katibougou, BP.06, Katibougou Koulikoro, Mali. \\ *Auteur correspondant ; E-mail: mldiarra.edu.ml@gmail.com; BP.1805. Tel.: (+223)76024656.
}

\section{RESUME}

Au Mali, le Gastrosédal qui est un Médicament Traditionnel Amélioré (MTA) produit à base de Vernonia kotschyana est utilisé pour traiter les gastrites et l'ulcère gastroduodénal. L'une des difficultés liées à la production de ce MTA est la disponibilité permanente et la mauvaise qualité de la matière première. L'objectif du présent travail était de cultiver et contrôler la qualité des plants de Vernonia kotschyana issus de différentes conditions de culture. L'étude, conduite en 2016 au Mali, a porté sur la culture de Vernonia kotschyana sous trois traitements de fertilisants (non apport de nutriment végétal $\left(\mathrm{T}_{0}\right)$, apport de nutriment à 5 tonnes/ha $\left(\mathrm{T}_{1}\right)$ et apport de nutriment à 10 tonnes/ha $\left(\mathrm{T}_{2}\right)$ ). Les plants obtenus ont été soumis à des analyses botanique et physicochimique par des méthodes spécifiques. Les résultats botaniques ont montré que la croissance et le diamètre au collet des plants avaient été stimulés à 180 jours après repiquage (JAR). Une différence significative avait été obtenue à 150 JAR pour la longueur et le diamètre des tubercules. Une différence significative a été aussi obtenue avec le rendement des tubercules. La microscopie a montré la présence de cellules polygonales allongées, de xylème spiralé, de fibres, de fragment de xylème pointu, de cristaux d'oxalate de calcium et de poil tecteur unicellulaire. Ce dernier élément n'a pas été retrouvé dans tous les échantillons. Quant aux résultats physicochimiques toutes les teneurs en eau des trois traitements à $180 \mathrm{JAR}$ étaient inférieures à $10 \%$. Cependant, le traitement $\mathrm{T}_{2}$ à $150 \mathrm{JAR}$ contenait plus de cendres chlorhydriques $(1,03 \%)$. Le traitement $\mathrm{T}_{1}$ à $150 \mathrm{JAR}$ contenait plus de substances extractibles par infusion (61\%). Les indices de mousse étaient tous élevés excepté celui de $\mathrm{T}_{0}$ à $120 \mathrm{JAR}$. Ces résultats ont révélé que Vernonia kotschyana mise en culture est de qualité et peut remplacer les plants sauvages; tout en assurant un approvisionnement suffisant en matière première pour la production du Gastrosédal et la préservation de l'environnement. (C) 2018 International Formulae Group. All rights reserved.

Mots clés : Vernonia kotschyana, culture, gastrites et ulcère gastroduodénal, plantes médicinales, Mali. 


\title{
Botanical and phytochemical characteristics of Vernonia kotschyana Sch. Beep. ex Walp. cultured and used in the treatment of gastritis and gastro duodenal ulcers in Mali
}

\begin{abstract}
In Mali, Gastrosedal which is an Improved Traditional Medicine (ITM) products from of Vernonia kotschyana is used to treat gastritis and gastro duodenal ulcers. One of difficulties linked to the production of this ITM is the permanent availability and the poor quality of raw material. The objective of this study was to cultivate and control the quality of Vernonia kotschyana's plants found from different conditions of culture. This study of the culture of Vernonia kotschyana, carried out in 2016 in Mali, focused on the culture of Vernonia kotschyana under three fertilizer treatments (without supplying plant nutrient $\left(\mathrm{T}_{0}\right)$, with nutrient supply at 5 tonnes / ha $\left(\mathrm{T}_{1}\right)$ and with nutrient supply at 10 tonnes / ha $\left(\mathrm{T}_{2}\right)$ ). Obtained plants were submitted to some botanical and physicochemical analysis by specific methods. The botanical results showed that plant growth and their collar diameter were stimulated at 180 days after transplanting (DAT). A significant difference was obtained at 150 DAT about tuber length and their diameter. A significant difference was also obtained with the yield of the tubers. Microscopy data showed the presence of elongated polygonal cells, spiral xylem, fibers, sharp xylem fragment, calcium oxalate crystals, and unicellular trichome. This last element was not found in all samples. About physicochemical, water contents were less than $10 \%$ in the three treatments at 180 DAT. However treatment $\mathrm{T}_{2}$ at $150 \mathrm{DAT}$ contained more hydrochloric ashes with $1.03 \%$. Treatment $\mathrm{T}_{1}$ at 150 DAT contained more extractable substances by infusion with $61 \%$. The foaming indexes were all high except for the one of $\mathrm{T}_{0}$ at 120 DAT. These results revealed that cultivated Vernonia kotschyana has a good quality and can replace the wild harvest by ensuring a proper supplying of raw material for the production of Gastrosedal and the preservation of the environment.
\end{abstract}

(C) 2018 International Formulae Group. All rights reserved.

Keywords: Vernonia kotschyana, culture, gastritis and gastro duodenal ulcers, medicinal plants, Mali.

\section{INTRODUCTION}

Le Gastrosédal est un Médicament Traditionnel Amélioré (MTA) caractérisé par des poils tecteurs unicellulaires, des fragments de cellules polygonales allongées, de fibres, de xylème spiralé et des cristaux d'oxalate de calcium (Ragusa et al., 2002).

Le Gastrosédal est produit à base des tubercules d'une Asteraceae appelée Vernonia kotschyana. Utilisé pour traiter les gastrites et l'ulcère gastroduodénal, il est sur la liste nationale des médicaments essentiels du Mali (Ministère de la Santé du Mali, 2005).

Sur le plan chimique, le Vernonia kotschyana contient de nombreux composés tels que les alcaloïdes, les terpénoïdes, les saponosides, les flavonoïdes, les protéines, les tanins, les polysaccharides, les dérivés triterpiniques et stéroïdique, les substances mucilagineuses, les oses, les composés réducteurs, les vernoniosides (Nergard et al., 2004 ; Coulibaly, 2007 ; Sanogo et al., 2010 ; Inngjerdingen et al., 2012).
Au Mali, il y'a eu quelques essais de culture de plantes médicinales dont le plus récent est celui de Vernonia kotschyana (Dembélé, 2009).

Le Gastrosédal est employé sous forme de poudre en infusion (Diallo et al., 2010). La poudre de racines (tubercules) de $V$. kotschyana épluchées est fine et de couleur blanc-cendre, elle est brun-noirâtre quand les racines ne sont pas épluchées. Elle a une saveur amère avec un arrière-goût sucré et mucilagineuse à la mastication. Ces racines s'écrasent entre les doigts en s'y collant.

Le Gastrosédal occupe une place très importante dans la thérapeutique au Mali. Il représentait le deuxième MTA le plus vendu en pharmacie après le Laxa-cassia à Kadiolo (Diallo et al., 2010).

Divers mécanismes d'action ont été suggérés pour le Gastrosédal tels que le revêtement et la protection de la muqueuse gastrique, la diminution du taux d'extrusion des cellules épithéliales et le maintien de 
l'intégrité épithéliale (Wangensteen et al, 2015).

Une des difficultés majeures de la production de ce MTA est la disponibilité et la qualité de la matière première qui provient des peuplements naturels de Kolokani et par culture. Cette disponibilité de la matière première est liée à des contraintes climatiques et anthropiques et la non maitrise de sa culture. La mauvaise qualité de la matière première est due à l'insuffisance d'étude botanique spécifique de la drogue surtout microscopique et phytochimique. C'est dans ce contexte que cette étude a été réalisée pour assurer un approvisionnement régulier et de qualité en matière première. Ainsi, cette étude à pour but de faire un essai de la culture de Vernonia kotschyana dans différentes conditions et contrôler la qualité des récoltes.

\section{MATERIEL ET METHODES}

L'essai de culture de Vernonia kotschyana a été réalisé dans le jardin potager de l'Institut Polytechnique Rural de Formation et de Recherche Appliquée (IPR/IFRA) de Katibougou et le contrôle de la qualité des échantillons cultivés a été réalisé au Laboratoire de Phytochimie du Département de Médecine Traditionnelle.

\section{Culture}

La méthode culturale a consisté à étudier la fertilisation organique de Vernonia kotschyana avec trois traitements. Le premier traitement a été le non apport de nutriment végétal $\left(\mathrm{T}_{0}\right)$, le deuxième traitement à l'apport de nutriment à 5 tonnes/hectare $\left(\mathrm{T}_{1}\right)$ et le troisième à l'apport de nutriment à 10 tonnes/hectare $\left(\mathrm{T}_{2}\right)$. Le matériel végétal a été constitué par des plants de Vernonia kotschyana obtenus après semis. Le nutriment végétal a été de la fiente de volaille. Le dispositif agronomique a été un bloc de Fisher avec 4 répétitions dans des planches de $10 \mathrm{~m}^{2}$ ( $5 \mathrm{~m}$ de long et $2 \mathrm{~m}$ de large) avec des allées de $1 \mathrm{~m}$ de large. Les semis ont été effectués le 11 janvier 2016 et les récoltes des tubercules ont été échelonnées du 13 juin au 13 août 2016, à raison d'environ 5 plants par parcelle élémentaire. Les caractères botaniques macroscopiques étudiés ont été la taille des plants, le diamètre au collet des plants, le diamètre et la longueur des tubercules, nombre de ramifications. Ces mesures ont été faites avec du mètre ruban et du pied à coulisse. Le rendement des tubercules à l'état frais et après séchage a été déterminé à l'aide d'une balance électronique. Ces données ont été analysées avec le logiciel STAT ITCF.

\section{Contrôle de qualité}

Les essais botanique et physicochimique ont consisté à la détermination des caractères organoleptiques, des éléments microscopiques, de la teneur en eau, de la teneur en cendres totales et chlorhydriques, des substances extractibles par les solvants et l'indice de mousse dans des échantillons de poudre de tubercules de Vernonia kotschyana. Le matériel a été constitué de poudre de tubercules de Vernonia kotschyana et des réactifs comprenant le réactif de Gazet du chatelier, l'eau, l'acide chlorhydrique à $10 \%$, l'éthanol 30 et $70 \%$ et l'éther de pétrole).

\section{Caractères organoleptiques de la poudre}

La détermination des caractères organoleptiques a consisté à déterminer la couleur, le goût, la taille (granulométrie) et l'odeur de la poudre de Vernonia kotschyana.

\section{Microscopie}

Dans un creuset en verre, une très petite quantité de poudre de Vernonia kotschyana a été versée avec une goutte du réactif de Gazet puis triturée. Une petite quantité de cette préparation, déposée entre une lame et une lamelle a été examinée au faible grossissement puis au fort grossissement.

\section{Teneur en eau}

Elle a été déterminée par la méthode pondérale en évaluant la perte de poids de la prise d'essai par dessiccation à l'étuve après 24 heures. Une quantité de 2 à $3 \mathrm{~g}$ de poudre de Vernonia kotschyana (masse prise d'essai) dans un verre de montre préalablement taré, a été placée dans l'étuve à $105{ }^{\circ} \mathrm{C}$ pendant 24 heures et la perte en poids a ensuite été déterminée. Cinq tests parallèles ont été effectués afin d'obtenir une moyenne. Le pourcentage en eau a été déterminé par les formules suivantes:

Masse prise d'essai $=$ masse avant étuve- tare 
Masse eau= masse avant étuve - masse après étuve

$$
\% \text { en eau }=\frac{\text { Masse eau }}{\text { Masse prise d'essai }} \times 100
$$

La tare est la masse du verre de montre. La masse avant étuve est celle de la prise d'essai avant dessiccation et celle du verre de montre. La masse après étuve est celle de la prise d'essai après dessiccation et celle du verre de montre.

\section{Cendres totales}

Trois prises d'essai de la poudre de Vernonia kotschyana utilisée pour la teneur en eau ont été pesées dans 3 creusets en porcelaine préalablement taré $(\mathrm{M})$. Après incinération au four à une température d'environ $600{ }^{\circ} \mathrm{C}$ pendant 6 heures, et refroidissement dans un dessiccateur, les masses (M') des creusets contenant les cendres ont été déterminées.

La masse des cendres totales (Mct) contenue dans le creuset et la masse de la prise d'essai (Mpe) sont déterminées par les formules suivantes :

$$
\begin{aligned}
& \text { Mct }=\mathrm{M}^{\prime}-\mathrm{T} \\
& \text { Mpe }=\mathrm{M}-\mathrm{T}
\end{aligned}
$$

La tare (T) est la masse du creuset en porcelaine. La masse de la prise d'essai (Mpe) est celle des prises d'essai utilisée pour la teneur en eau avant incinération moins celle des creusets. La masse des cendres totales (Mct) est celle des prises d'essai utilisée pour la teneur en eau après incinération moins celle des creusets.

Le pourcentage des cendres totales $(\% \mathrm{Ct})$ est calculé par la formule suivante:

$$
\% \mathrm{Ct}=\frac{\mathrm{Mct}}{\mathrm{Mpe}} \mathrm{x}
$$

\section{Cendres chlorhydriques}

Ces cendres sont obtenues en faisant bouillir les cendres totales dans de l'acide chlorhydrique à $10 \%$. Les cendres totales préalablement obtenues ont été bouillies dans $20 \mathrm{ml}$ d'acide chlorhydrique et filtrées sur du papier sans cendre. Ce papier a été transféré dans un creuset sec déjà taré de masse $\mathrm{M}$. L'ensemble est pesé (M'), incinéré et repesé $\left(\mathrm{M}^{\prime \prime}\right)$.

La masse des cendres chlorhydriques (MCc) est donnée par la formule suivante : $\mathrm{MCc}=\mathrm{M}^{\prime}-\mathrm{M}^{\prime}$,

Le pourcentage des cendres chlorhydriques (\%Cc) est calculé par la formule suivante :

$$
\% C c=\frac{M C c}{\sum M p e} \times 100
$$

$\sum$ Mpe est la somme des masses de prise d'essai pour la détermination de la teneur en eau.

\section{Substances extractibles}

Elles sont déterminées à partir de $1 \mathrm{~g}$ de la poudre de Vernonia kotschyana avec 20 $\mathrm{ml}$ du solvant. Après filtration, le filtrat a été évaporé à sec dans un creuset préalablement taré. Soit $\left(\mathrm{M}_{1}\right)$ la masse du creuset vide et $\left(\mathrm{M}_{2}\right)$ la masse du creuset avec l'extrait sec. Le pourcentage de substances extractibles a été calculé par la formule :

$\%$ substances extractibles par le solvant $(\mathrm{g})=\frac{m_{2}-M_{1}}{1} \times 100$

Les solvants utilisés étaient l'eau après une infusion (5\%), l'éthanol $30 \%$ et $70 \%$ et l'éther de pétrole après une macération.

\section{Indice de mousse}

Il a été déterminé en pesant $1 \mathrm{~g}$ de la poudre de Vernonia kotschyana, mélangé à $100 \mathrm{ml}$ d'eau distillée. Le mélange est ensuite porté à l'ébullition pendant $15 \mathrm{mn}$. Après l'ébullition, la solution est filtrée puis partagée dans 10 tubes numérotés de 1 à 10 . Le partage est fait comme suite : $1 \mathrm{ml}$ dans le tube $\mathrm{n}^{\circ} 1,2$ $m l$ dans le tube $n^{\circ} 2$ et ainsi de suite jusqu'au tube $n^{\circ} 10$. L'ensemble des tubes est complété à $10 \mathrm{ml}$ avec de l'eau distillée. Ensuite on agite le tube dans le sens de la longueur 2 fois/seconde pendant 15 secondes et on attend 15 minutes pour mesurer la hauteur de la mousse persistante. Le tube avec la hauteur de $1 \mathrm{~cm}$ de mousse correspond au tube de l'indice de mousse.

L'indice de mousse (I) est calculé avec la formule suivante: $I=\frac{1000}{N}$ 
Si on dilue à $1 / 2 \mathrm{I}$ devient $\mathrm{I}=\frac{1000}{\frac{\mathrm{N}}{\frac{1}{2}}}=\frac{2000}{\mathrm{~N}}$. $\mathrm{N}$ est le numéro du tube.

\section{RESULTATS}

Les résultats obtenus ont montré que la fertilisation n'a pas eu de différence significative entre les moyennes des traitements sur la taille des plants (Tableau 1). Elle a néanmoins stimulé la croissance des plants avec une moyenne de 108,93 $\mathrm{cm}$ pour $\mathrm{T}_{1}$ et $112,22 \mathrm{~cm}$ pour $\mathrm{T}_{2}$ à $180 \mathrm{JAR}$. Ce même résultat a été obtenu avec le diamètre au collet des plants qui a été proportionnel à la taille des plants (Tableau 2). Une différence significative a été observée entre les moyennes de la longueur $\left(25,64 \mathrm{~cm}\right.$ pour $\mathrm{T}_{2}$ contre $20,16 \mathrm{~cm}$ pour $\mathrm{T}_{0}$ ) et le diamètre des tubercules $\left(3,73 \mathrm{~cm}\right.$ pour $\mathrm{T}_{2}$ contre $3,00 \mathrm{~cm}$ pour $\mathrm{T}_{0}$ ) à 150 JAR (Tableau 3). Une différence significative a été observée sur la moyenne du nombre de ramifications entre les traitements $\left(8,19\right.$ pour $\mathrm{T}_{2}$ contre 4,43 pour $\left.\mathrm{T}_{0}\right)$ à 150 JAR (Tableau 4). Aucune différence significative n'a été observée entre les moyennes des traitements avec le nombre d'inflorescences (Tableau 4). La différence observée entre le rendement des tubercules à l'état frais et après séchage des différents traitements était significative à 150 JAR (Tableau 5). Le rendement le plus élevé; 2,96 tonnes/ha a été obtenu avec $\mathrm{T}_{1}$ contre 1,59 tonnes/ha pour $\mathrm{T}_{0}$.

Les essais botaniques ont montré que tous les échantillons présentaient les caractères organoleptiques décrits par les études du Département de Médecine Traditionnelle sur Vernonia kotschyana. Des cellules polygonales allongées, de xylème spiralé, de fibres, de fragment de xylème pointu et de cristaux d'oxalate de calcium ont été retrouvés dans tous les traitements (Figures 2, 3, 4 et 5). Seul $\mathrm{T}_{1}$ à $150 \mathrm{JAR}$ a montré la présence de poils tecteurs unicellulaires (Figure 1). Les teneurs en eau dans les trois traitements étaient inférieures à $10 \%$ à $180 \mathrm{JAR}$ (Tableau 6). Le traitement $\mathrm{T}_{1}$ avec $61 \%$ à 150 JAR contenait plus de substances extractibles par l'eau après infusion que les autres traitements (Tableau 6). Les cendres totales étaient approximativement les mêmes. Pour les cendres chlorhydriques, $\mathrm{T}_{2}$ avec $1,03 \%$ à 150 JAR contenait beaucoup plus de matières siliceuses que les autres traitements (Tableau 7). Le traitement $\mathrm{T}_{0}$ à $150 \mathrm{JAR}$ contenait 2 à 3 fois plus de substances extractibles par l'éthanol à 30\%. Avec l'éthanol à $70 \%$ tous les traitements à 180 JAR contenaient 2 à 3 fois plus de substances extractibles que les traitements de 120 et 150 JAR (Tableau 8). Les indices en saponosides étaient tous élevés excepté $\mathrm{T}_{0}$ à 120 JAR (Tableau 9).

Tableau 1 : Taille des plants de Vernonia kotschyana après application de la fiente de volaille.

\begin{tabular}{lcccccc}
\hline Traitements & \multicolumn{5}{c}{ Taille des plants $(\mathbf{c m})$} \\
\hline Fréquences & $\mathbf{5 0}$ JAR & 60 JAR & 70 JAR & 120 JAR & 150 JAR & 180 JAR \\
$\mathrm{T}_{0}$ & 31,83 & 44,57 & 54,02 & 79,95 & 88,38 & 95,74 \\
$\mathrm{~T}_{1}$ & 42,65 & 54,95 & 59,28 & 84,97 & 93,66 & 108,93 \\
$\mathrm{~T}_{2}$ & 47,85 & 63,95 & 64,98 & 90,83 & 102,91 & 112,22 \\
$\mathrm{MG}$ & 40,78 & 54,49 & 59,43 & 85,25 & 94,98 & 105,63 \\
Proba & 0,0874 & 0,0607 & 0,4006 & 0,1127 & 0,2368 & 0,2847 \\
\hline Sign & $\mathrm{NS}$ & $\mathrm{NS}$ & $\mathrm{NS}$ & $\mathrm{NS}$ & $\mathrm{NS}$ & $\mathrm{NS}$ \\
$\mathrm{CV} \%$ & 20,7 & 16,5 & 17,8 & 7,1 & 11,4 & 13,2 \\
\hline
\end{tabular}

$\mathrm{MG}=$ moyennes Générales, Proba $=$ probabilité, Sign $=$ signification, $\mathrm{CV}=$ coefficient de variance, $\mathrm{NS}=$ non significative, $\mathrm{JAR}=$ jour après repiquage. 
Tableau 2 : Diamètre au collet des plants de Vernonia kotschyana après application de la fiente de volaille.

\begin{tabular}{lcccccc}
\hline Traitements & \multicolumn{7}{c}{ Diamètre au collet des plants (cm) } \\
\hline Fréquences & $\mathbf{5 0}$ JAR & 60 JAR & 70 JAR & 120 JAR & 150 JAR & 180 JAR \\
$\mathrm{T}_{0}$ & 0,55 & 0,71 & 1,02 & 1,96 & 1,74 & 1,79 \\
$\mathrm{~T}_{1}$ & 0,62 & 0,85 & 1,28 & 2,21 & 2,25 & 2,30 \\
$\mathrm{~T}_{2}$ & 0,75 & 0,94 & 1,31 & 2,37 & 2,21 & 2,37 \\
$\mathrm{MG}$ & 0,64 & 0,83 & 1,21 & 2,18 & 2,07 & 2,15 \\
Proba & 0,1495 & 0,1327 & 0,3475 & 0,5040 & 0,0790 & 0,4056 \\
Sign & $\mathrm{NS}$ & $\mathrm{NS}$ & $\mathrm{NS}$ & $\mathrm{NS}$ & $\mathrm{NS}$ & $\mathrm{NS}$ \\
CV\% & 20,0 & 16,4 & 23,7 & 21,0 & 13,7 & 28,4 \\
\hline
\end{tabular}

MG = moyennes Générales, Proba = probabilité, Sign = signification, $\mathrm{CV}=$ coefficient de variance, NS = non significative, $\mathrm{JAR}=$ jour après repiquage.

Tableau 3: Longueur et diamètre des tubercules des plants de Vernonia kotschyana après application de la fiente de volaille.

\begin{tabular}{lcccccc}
\hline Traitements & \multicolumn{3}{c}{ Longueur des tubercules $(\mathbf{c m})$} & \multicolumn{3}{c}{ Diamètre des tubercules $(\mathbf{c m})$} \\
\hline Fréquences & 120 JAR & $\mathbf{1 5 0}$ JAR & $\mathbf{1 8 0 ~ J A R}$ & $\mathbf{1 2 0}$ JAR & 150 JAR & $\mathbf{1 8 0}$ JAR \\
$\mathrm{T}_{0}$ & 19,35 & $20,16 \mathrm{~B}$ & 22,76 & 3,43 & $3,00 \mathrm{~B}$ & 3,10 \\
$\mathrm{~T}_{1}$ & 22,07 & $23,20 \mathrm{AB}$ & 23,28 & 3,85 & $3,89 \mathrm{~A}$ & 3,75 \\
$\mathrm{~T}_{2}$ & 22,49 & $25,64 \mathrm{~A}$ & 25,55 & 3,99 & $3,73 \mathrm{~A}$ & 3,91 \\
$\mathrm{MG}$ & 21,30 & 23,00 & 23,86 & 3,75 & 3,54 & 3,59 \\
Proba & 0,2854 & 0.0200 & 0,7718 & 0,3299 & 0,0250 & 0,3245 \\
Sign & $\mathrm{NS}$ & $\mathrm{S}$ & $\mathrm{NS}$ & $\mathrm{NS}$ & $\mathrm{S}$ & $\mathrm{NS}$ \\
$\mathrm{CV} \%$ & 12,8 & 84 & 23,8 & 13,3 & 9,9 & 20,4 \\
\hline
\end{tabular}

$\mathrm{MG}=$ moyennes Générales, Proba = probabilité, Sign = signification, $\mathrm{CV}=$ coefficient de variance, $\mathrm{NS}$ = non significative, $\mathrm{S}$ $=$ significative, $\mathrm{JAR}=$ jour après repiquage, $\mathrm{A}, \mathrm{B}, \mathrm{AB}=$ groupes homogènes avec $\mathrm{A}>\mathrm{AB}>\mathrm{B}$.

Tableau 4 : Nombre de ramifications et d'inflorescences des plants de Vernonia kotschyana après application de la fiente de volaille.

\begin{tabular}{lcccccc}
\hline Traitements & \multicolumn{3}{c}{ Nombre de ramifications } & \multicolumn{3}{c}{ Nombre d'inflorescences } \\
\hline Fréquences & 120 JAR & 150 JAR & 180 JAR & 120 JAR & 150 JAR & 180 JAR \\
$\mathrm{T}_{0}$ & 6,71 & $4,43 \mathrm{~B}$ & 2,93 & 8,90 & 1,68 & 0,72 \\
$\mathrm{~T}_{1}$ & 10,47 & $6,26 \mathrm{AB}$ & 5,93 & 12,07 & 5,31 & 2,39 \\
$\mathrm{~T}_{2}$ & 11,35 & $8,19 \mathrm{~A}$ & 6,50 & 12,76 & 7,88 & 4,70 \\
MG & 9,51 & 6,30 & 5,12 & 11,25 & 4,96 & 2,60 \\
Proba & 0,0870 & 0,0118 & 0,4122 & 0,2491 & 0,1374 & 0,2567 \\
Sign & $\mathrm{NS}$ & $\mathrm{S}$ & $\mathrm{NS}$ & $\mathrm{NS}$ & $\mathrm{NS}$ & $\mathrm{NS}$ \\
CV\% & 26,7 & 18,5 & 73,7 & 27,6 & 75,0 & 117,0 \\
\hline
\end{tabular}

MG = moyennes Générales, Proba $=$ probabilité, Sign $=$ signification, $\mathrm{CV}=$ coefficient de variance, $\mathrm{NS}=$ non significative, $\mathrm{S}$ $=$ significative, $\mathrm{JAR}=$ jour après repiquage, $\mathrm{A}, \mathrm{B}, \mathrm{AB}=$ groupes homogènes avec $\mathrm{A}>\mathrm{AB}>\mathrm{B}$. 
Tableau 5: Rendement frais et après séchage des tubercules de Vernonia kotschyana après application de la fiente de volaille.

\begin{tabular}{lcccccc}
\hline Traitements & \multicolumn{5}{c}{ Rendement des tubercules } \\
\cline { 2 - 7 } & \multicolumn{5}{c}{ Frais (t/ha) } & \multicolumn{5}{c}{ Après séchage (t/ha) } \\
\hline Fréquences & $\mathbf{1 2 0}$ JAR & $\mathbf{1 5 0}$ JAR & $\mathbf{1 8 0 ~ J A R}$ & $\mathbf{1 2 0}$ JAR & $\mathbf{1 5 0}$ JAR & $\mathbf{1 8 0}$ JAR \\
$\mathrm{T}_{0}$ & 2,33 & $1,59 \mathrm{~B}$ & 2,57 & 0,79 & $0,66 \mathrm{~B}$ & 0,68 \\
$\mathrm{~T}_{1}$ & 3,05 & $2,96 \mathrm{~A}$ & 3,30 & 1,11 & $1,08 \mathrm{~A}$ & 0,90 \\
$\mathrm{~T}_{2}$ & 3,25 & $2,77 \mathrm{~A}$ & 4,02 & 1,13 & $1,04 \mathrm{~A}$ & 1,04 \\
$\mathrm{MG}$ & 2,88 & 2,44 & 3,29 & 1,01 & 0,93 & 0,87 \\
Proba & 0,4259 & 0,0132 & 0,5817 & 0,1799 & 0,0286 & 0,6262 \\
Sign & $\mathrm{NS}$ & $\mathrm{S}$ & $\mathrm{NS}$ & $\mathrm{NS}$ & $\mathrm{S}$ & $\mathrm{NS}$ \\
CV\% & 33,7 & 19,4 & 56,8 & 24,4 & 18,7 & 57,7 \\
\hline
\end{tabular}

$\mathrm{t}=$ tonne, ha $=$ hectare, $\mathrm{MG}=$ moyennes Générales, Proba = probabilité, Sign = signification, $\mathrm{CV}=$ coefficient de variance, $\mathrm{NS}=$ non significative, $\mathrm{S}=$ significative, $\mathrm{JAR}=$ jour après repiquage, $\mathrm{A}, \mathrm{B},=$ groupes homogènes avec $\mathrm{A}>\mathrm{B}$.
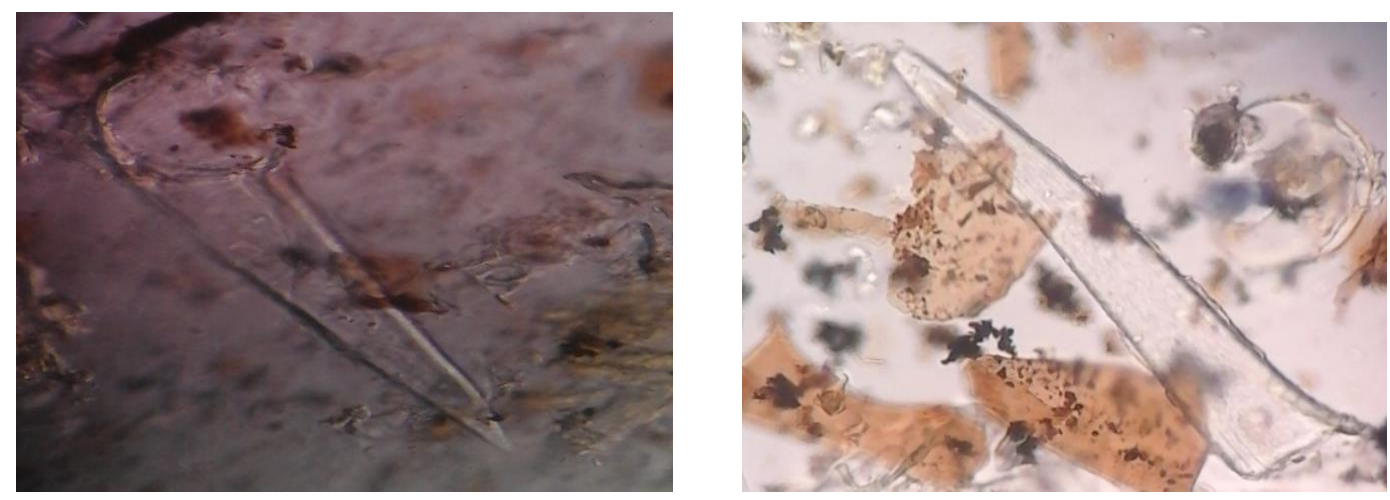

Figure 1 : Poil tecteur unicellulaire de poudre de Vernonia kotschyana.
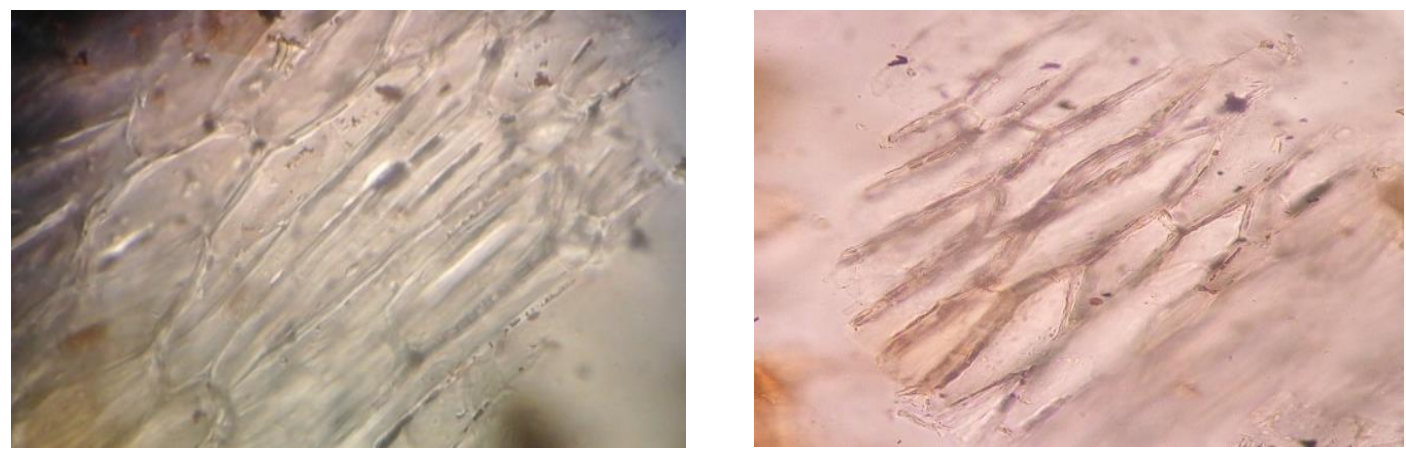

Figure 2 : Cellules polygonales allongées de poudre de Vernonia kotschyana. 

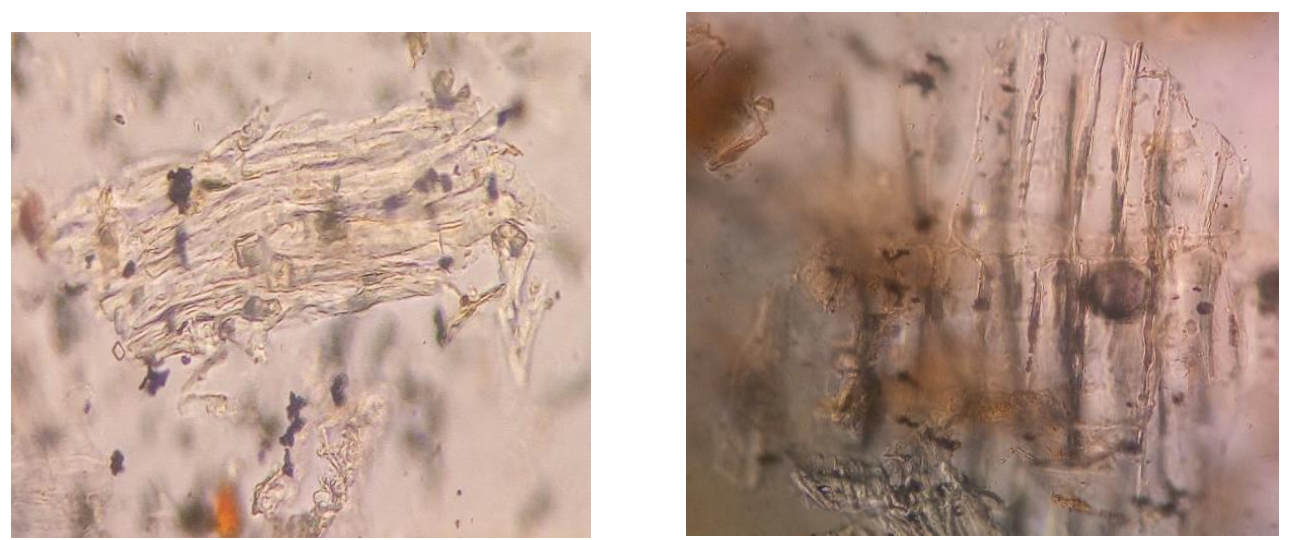

Figure 3 : Fibres de poudre de Vernonia kotschyana.
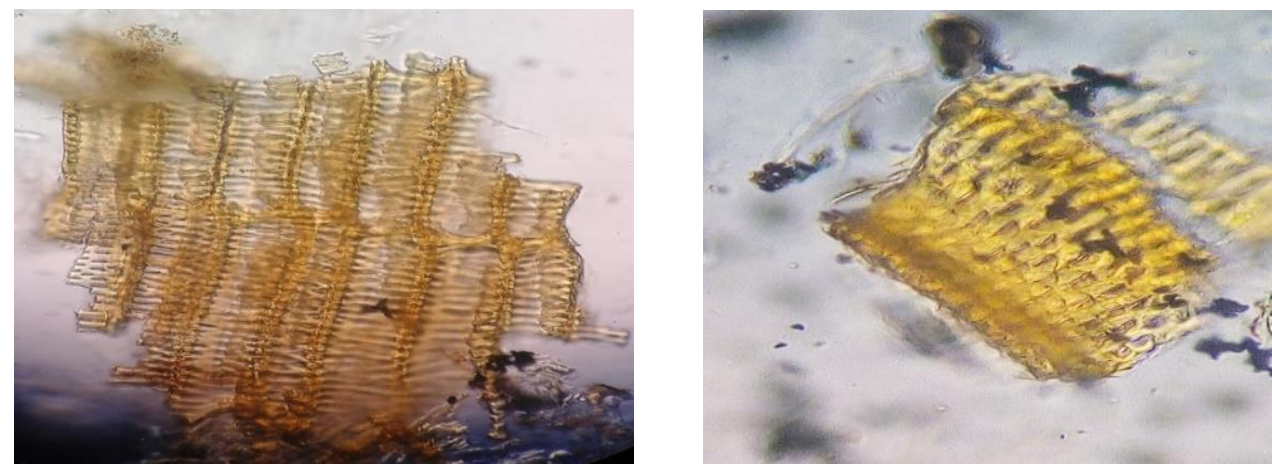

Figure 4 : Xylème spiralé et fragment de xylème pointu de poudre de Vernonia kotschyana.

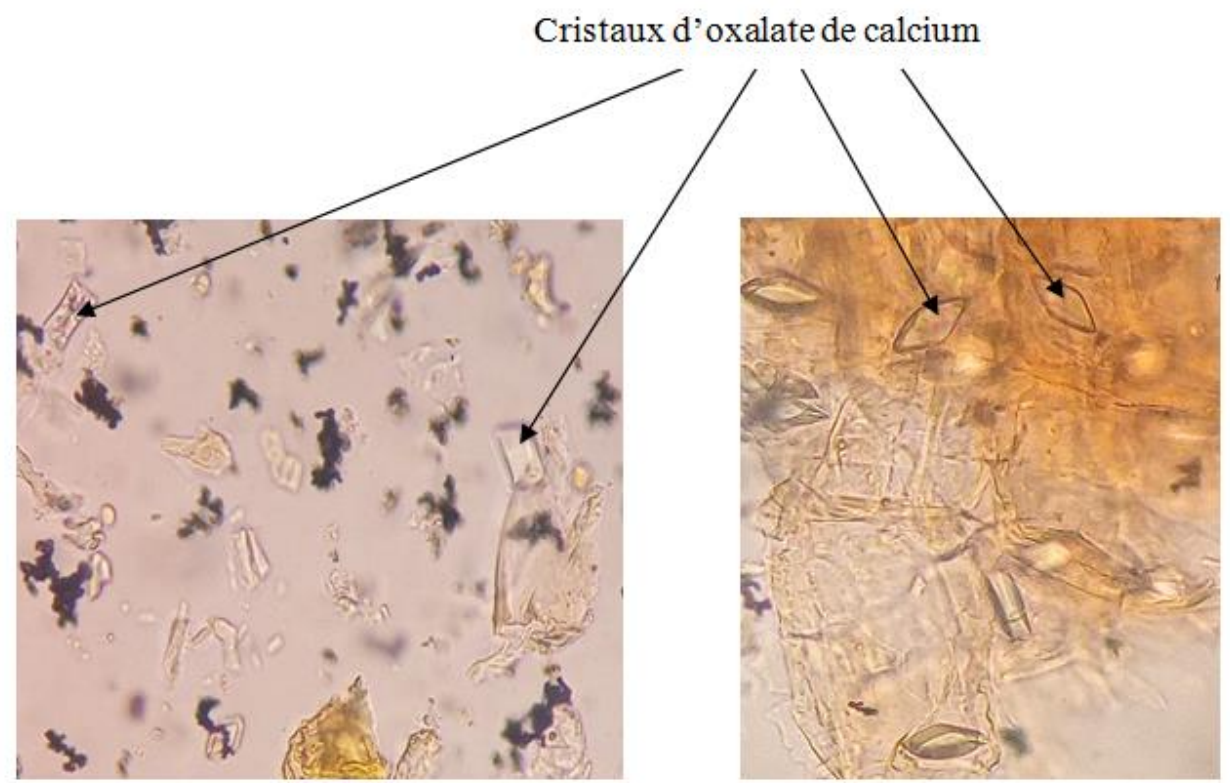

Figure 5 : Cristaux d'oxalate de calcium de poudre de Vernonia kotschyana. 
Tableau 6 : Teneur en eau et de substances extraites par l'eau après infusion des échantillons de Vernonia kotschyana après application de la fiente de volaille.

\begin{tabular}{lccc}
\hline Fréquences & Traitements & Teneur en eau (\%) & Teneur de substances extraites par l'eau (\%) \\
\hline \multirow{2}{*}{ 120 JAR } & $\mathrm{T}_{0}$ & 10,85 & 55 \\
& $\mathrm{~T}_{1}$ & 10,31 & 55 \\
& $\mathrm{~T}_{2}$ & 12,74 & 59 \\
\hline \multirow{2}{*}{ 150 JAR } & $\mathrm{T}_{0}$ & 11,24 & 55 \\
& $\mathrm{~T}_{1}$ & 12,25 & 61 \\
& $\mathrm{~T}_{2}$ & 10,97 & 54 \\
\hline \multirow{2}{*}{ 180 JAR } & $\mathrm{T}_{0}$ & 3,67 & 41 \\
& $\mathrm{~T}_{1}$ & 4,22 & 29 \\
& $\mathrm{~T}_{2}$ & 5,62 & 50 \\
\hline
\end{tabular}

Tableau 7 : Teneur en cendre totale $(\mathrm{Ct})$ et en cendres chlorhydriques $(\mathrm{Cc})$ des échantillons de Vernonia kotschyana après application de la fiente de volaille.

\begin{tabular}{lccc}
\hline Fréquences & Traitements & Teneur en Ct $\mathbf{( \% )}$ & Teneur en Cc (\%) \\
\hline \multirow{2}{*}{ 120 JAR } & $\mathrm{T}_{0}$ & 6,50 & 0,74 \\
& $\mathrm{~T}_{1}$ & 4,99 & 0,73 \\
& $\mathrm{~T}_{2}$ & 5,11 & 0,45 \\
\hline \multirow{3}{*}{ 150 JAR } & $\mathrm{T}_{0}$ & 4,94 & 0,89 \\
& $\mathrm{~T}_{1}$ & 4,55 & 0,89 \\
& $\mathrm{~T}_{2}$ & 3,89 & 1,03 \\
\hline \multirow{2}{*}{ 180 JAR } & $\mathrm{T}_{0}$ & 5,36 & 0,33 \\
& $\mathrm{~T}_{1}$ & 5,09 & 0,25 \\
& $\mathrm{~T}_{2}$ & 4,60 & 0,49 \\
\hline
\end{tabular}

JAR $=$ jour après repiquage, $\mathrm{Ct}=$ cendres totales, $\mathrm{Cc}=$ cendres chlorhydriques.

Tableau 8 : Substances extractibles par l'éthanol à $30 \%$ et $70 \%$ des échantillons de Vernonia kotschyana après application de la fiente de volaille.

\begin{tabular}{lccc}
\hline Fréquences & Traitements & $\begin{array}{c}\text { Teneur en substances } \\
\text { extraites, éthanol (30\%) }\end{array}$ & $\begin{array}{c}\text { Teneur en substances } \\
\text { extraites, éthanol (70\%) }\end{array}$ \\
\hline \multirow{2}{*}{ 120 JAR } & $\mathrm{T}_{0}$ & 13 & 11 \\
& $\mathrm{~T}_{1}$ & 3 & 9 \\
& $\mathrm{~T}_{2}$ & 14 & 11 \\
\hline \multirow{2}{*}{ 150 JAR } & $\mathrm{T}_{0}$ & 35 & 15 \\
& $\mathrm{~T}_{1}$ & 6 & 11 \\
& $\mathrm{~T}_{2}$ & 13 & 16 \\
\hline \multirow{2}{*}{ 180 JAR } & $\mathrm{T}_{0}$ & 13 & 27 \\
& $\mathrm{~T}_{1}$ & 9 & 31 \\
& $\mathrm{~T}_{2}$ & 1 & 30 \\
\hline
\end{tabular}

$\mathrm{JAR}=$ jour après repiquage. 
Tableau 9: Indices de mousse des échantillons de Vernonia kotschyana après application de la fiente de volaille.

\begin{tabular}{lcc}
\hline Fréquences & Traitements & Indice de mousse \\
\hline \multirow{2}{*}{ 120 JAR } & $\mathrm{T}_{0}$ & 333,33 \\
& $\mathrm{~T}_{1}$ & 500 \\
& $\mathrm{~T}_{2}$ & 500 \\
\hline \multirow{2}{*}{ 150 JAR } & $\mathrm{T}_{0}$ & 500 \\
& $\mathrm{~T}_{1}$ & 500 \\
& $\mathrm{~T}_{2}$ & 500 \\
\hline \multirow{2}{*}{ 180 JAR } & $\mathrm{T}_{0}$ & 400 \\
& $\mathrm{~T}_{1}$ & 400 \\
& $\mathrm{~T}_{2}$ & 500 \\
\hline
\end{tabular}

$\mathrm{JAR}=$ jour après repiquage.

\section{DISCUSSION}

La culture de Vernonia kotschyana avec sans apport de nutriment, avec apport de nutriment à 5 tonnes/ha et à 10 tonnes/ha n'a pas montré de différence significative de façon générale; bien que les plus grandes valeurs de taille de plant, de diamètre au collet et du nombre d'inflorescence aient été obtenues avec le traitement $T_{2}$. Une différence significative avait été signalée par Dembélé (2009) avec la taille et le diamètre au collet des plants pour différents traitements.

Les meilleurs rendements après récolte des tubercules ont été obtenus avec l'apport maximum du fertilisant $\left(\mathrm{T}_{2}\right)$ même si la différence n'était pas significative avec les autres traitements. Ce résultat aussi est similaire à celui de Dembélé (2009), qui avait obtenu un rendement plus élevé avec une densité de peuplement plus important.

Une différence significative a été observée sur la moyenne des longueurs des tubercules et le nombre de ramifications à 150 JAR avec la plus grande valeur pour le traitement $T_{2}$. Ce même résultat a été obtenu avec le diamètre des tubercules et le rendement des récoltes à l'état frais et après séchage des tubercules avec cette fois la plus grande valeur pour le traitement $\mathrm{T}_{1}$.

La texture des tubercules issus du traitement $\mathrm{T}_{1}$ était moins ligneuse que ceux du traitement $T_{2}$ du fait de l'état de maturation plus avancé lié à la fertilisation plus importante.
Les éléments microscopiques observés avaient été obtenu par Ragusa et al. (2002) exceptés les fragments de xylème pointu décrits pour la première fois chez Vernonia kotschyana. Cependant, les poils tecteurs unicellulaires n'ont pas été retrouvés dans tous les échantillons contrairement à l'étude de Ragusa et al. Donc ces poils ne constituent pas un caractère microscopique constant pour le contrôle de qualité botanique de Vernonia kotschyana.

Le traitement $\mathrm{T}_{0}$ à $150 \mathrm{JAR}$ contenait 2 à 3 fois plus de substances extractibles par l'éthanol à $30 \%$ que les autres traitements. Avec l'éthanol à $70 \%$, elles étaient plus importantes avec tous les traitements à 180 JAR.

Les teneurs en cendres totales n'ont pas montré de différence significative entre les traitements. Les cendres chlorhydriques étaient relativement faibles.

La teneur en eau est inférieure à $10 \%$ à 180 JAR, les échantillons de 120 JAR et 150 JAR qui n'étaient pas découpés en petits morceaux n'avaient pas été bien séchés.

A part $\mathrm{T}_{0}$ à $120 \mathrm{JAR}$, les indices de mousses n'ont pas montré de différence significative entre les traitements.

\section{Conclusion}

L'espèce Vernonia kotschyana Sch. Bip. ex Walp. S'est bien prêtée à la culture dans les conditions expérimentales. Les plus grandes valeurs des caractères 
macromorphologiques (taille des plants, le diamètre au collet des tiges, la longueur et le diamètre des tubercules et nombres d'inflorescences) ont été obtenues avec le traitement $T_{2}$. Cette étude a permis de mettre à la disposition du monde scientifique d'importantes informations pour l'amélioration des itinéraires techniques dans la démarche vers une éventuelle domestication de la plante et une sauvegarde de cette ressource naturelle en utilisant un nutriment végétal.

\section{CONFLIT D'INTERETS}

Il n'y a pas eu de conflit d'intérêts.

\section{CONTRIBUTIONS DES AUTEURS}

MLD a conçu, conduit l'étude et participé à la rédaction de l'article. AD BLC et $\mathrm{AT}$ ont participé à la rédaction de l'article. $\mathrm{RS}, \mathrm{MT}$, DD et $\mathrm{KN}$ ont relu et corrigé l'article.

\section{REMERCIEMENTS}

Les auteurs remercient le personnel du laboratoire de Botanique et Biodiversité, de l'herbier Dakar du Département de Biologie Végétale de la Faculté des Sciences et Technique de l'UCAD, du Département de Médecine Traditionnelle, du Laboratoire d'Ethnobotanique de l'Institut Polytechnique Rural de Formation et de Recherche Appliquée (IPR/IFRA) de Katibougou.

\section{REFERENCES}

Ragusa S, Sanogo R, Germanó MP, Iauk L, De Pasquale R. 2002. Micromorphological investigations on Vernonia kotschyana Sch. Bip., Asteraceae IOCD International Symposium, Bamako, Mali, 6-8 March 2002

Nergard CS, Diallo D, Michaelsen TE, Malterud KE, Kiyohara H, Matsumoto
T, Yamada H, Paulsen BS. 2004. Isolation, partial characterisation and immunomodulating activities of polysaccharides from Vernonia kotschyana Sch. Bip. ex Walp. Journal of Ethnopharmacology, 91: 141-152. DOI : 10.1016/j.jep.2003.12.007

Ministère de la Santé du Mali. 2005. Formulaire thérapeutique national. Bamako, p. 477.

Coulibaly A. 2007. Etude de la phytochimie et des activités biologiques des feuilles de Vernonia kotschyana Sch.Bip (Asteraceae). FMPOS, Université Bamako, Bamako, p. 137.

Dembélé S. 2009. La réponse physiologique de Vernonia kotschyana (Sch. Bip.) à trois techniques culturales: le billonnage, la densité de peuplement et la dose d'engrais. Mémoire de D.E.A. Université de Ouagadougou, Ouagadougou, p. 49.

Sanogo R, Germano MP, De Tommasi N, Pizza C, Aquino RP. 2010. Vernoniosides and Androstane Glycosides from Vernonia kotschyana. ChemInform, DOI: 10.1002/chin.199813172

Inngjerdingen KT, Meskini S, Austarheim I, Ballo N, Inngjerdingen M, Michaelsen TE, Diallo D, Paulsen BS. 2012. Chemical and biological characterization of polysaccharides from wild and cultivated roots of Vernonia kotschyana. Journal of Ethnopharmacology, 139: 350- 358. DOI: $10.1016 /$ j.jep.2011. 10.044

Wangensteen H, Diallo D, Paulsen BS. 2015. Medicinal plants from Mali: Chemistry and biology. Journal of Ethnopharmacology, 176: 429-437. DOI: $\quad$ http://dx.doi.org/10.1016/ j.jep.2015.11.030. 